\title{
Intestinal dysbiosis and hormonal neuroendocrine secretion in the fibromyalgic patient
}

\author{
Giovanni Tomaselloa, Margherita Mazzola ${ }^{\mathrm{a}, \mathrm{b}}$, Vincenzo Boscoc, Giulia Tomasellod, Provvidenza Damianib,e, \\ Emanuele Sinagra ${ }^{b, f}$, Francesco Carini ${ }^{a, b, e}$
}

Fibromyalgia is a rheumatic syndrome and its pathogenesis is controversial. The recent literature has placed considerable attention on the link between alteration of the intestinal microbiota and fibromyalgia, emphasizing the close connection between the neuroenteric system and the CNS. This study aims to evaluate the probable relationship between intestinal dysbiosis and altered secretion of hormones and vitamins such as cortisol, serotonin, Vitamin D and thyroid hormones in a patient with fibromyalgia.

Key words: microbiota, fibromyalgia, hormones, dysbiosis, serotonin, cortisol, vitamin D

Received: April 6, 2018; Accepted with revision: August 30, 2018; Available online: September 11, 2018

https://doi.org/10.5507/bp.2018.051

${ }^{a}$ Department of Experimental Biomedicine and Clinical Neuroscience, Section of Human Anatomy, (BIONEC), School of Medicine and Surgery, University of Palermo, Palermo, Italy

${ }^{b}$ Euro-Mediterranean Institute of Science and Technology (IEMEST), Palermo, Italy

'School of Medicine, Palermo, Italy

${ }^{d}$ Kore University, Enna, Italy

eUniversitary Hospital, AOUP "P. Giaccone", Palermo, Italy

${ }^{f}$ Giglio Hospital, Cefalu, Italy

Corresponding author: Margherita Mazzola, e-mail: margheritamazzola@hotmail.it

\section{INTRODUCTION}

The relationship between altered microbiota and the subject of intestinal and extra intestinal diseases is a topic of current interest. New research techniques, numerous studies and an increasingly widespread scientific interest, have permitted us to understand better how the etiopathogenesis of some diseases may have as an initial stage a change at the level of the intestinal microbiota. The gut, which has been studied for years exclusively as a peripheral organ, has been the subject of several studies in recent decades allowing us to study the intestinal nerve network which given its size and mode of operation is now referred to as the "second brain", capable of interacting with the central nervous system (CNS) via the vagus nerve. The close mutual connection between the intestines and the CNS allows for one organ to modify the other and for this reason, a balance needs to be maintained at the level of this axis.

Fibromyalgia (FM) is a rheumatic syndrome characterized by diffuse musculoskeletal pain associated with sleep disorders, non-restorative sleep, fatigue, hyposthenia, headache, anxiety and depressive problems. Its pathogenesis is controversial as the etiopathogenetic mechanisms are numerous (sympathetic hyperactivity, alteration of neurotransmitters in CNS, neurogenic and mitochondrial changes genetic predisposition).

The alteration of intestinal bacteria and possible intestinal hyperperpermeability could have a surprising primary role in FM etiology. Starting from the obvious connection between the appearance of the FM and altered microbiota, this paper examines how the microbiota is able to alter hormone secretion, through the circle of lipopolysaccharide (LPS), the influence of the microbiota on the hypothalamic-pituitary-adrenal (HPA) and hypothalamus - hypophysis axes. Confirmation of the type of interactions described here, may revolutionize the way we look at and treat fibromyalgia and other chronic diseases.

\section{Gut microbiota}

In our body, a large number of viruses, bacteria and unicellular organisms coexist. The totality of microorganisms that coexist peacefully with the human host, is called the "microbiota". Its alteration, indicated by the term of dysbiosis, predisposes the individual to diseases, such as inflammatory bowel disease (IBD) and, according to recent studies, fibromyalgia. The human microbiota consists of at least $10^{14}$ bacterial cells with about 500-1000 different species divided into colonies ${ }^{1,2}$. In the intestinal tract, in particular, there are four main bacterial families (Firmicutes, Bacteroidetes, Proteobacteria and Actinobacteria) although there is a unique combination of species in each individual ${ }^{3,4}$. The microbiota in the GIT has a fundamental role in determining pathological states and in the maintenance of the health status of the individual. In fact, a normal microbiota participates in host nutrient metabolism (carbohydrates, vitamins, peptides, proteins), in the synthesis of short-chain fatty acid (SCFAs), in xenobiotic and drug metabolism, in maintenance of structural integrity of the gut mucosal barrier, 
in the regulation of the systemic immune response by influencing the functionality of the associated intestinal lymphoid tissue (GALT) and preventing the proliferation of pathogenic bacteria ${ }^{5}$. The microbiota, if in equilibrium, and therefore in eubiosis, contributes to a healthy functioning of the intestine and the whole organism. In the case of dysbiosis, however, there is an alteration of the intestinal flora quantitatively and qualitatively, and this can lead to the development of various diseases owing to the uncontrolled multiplication of harmless bacteria, which then become dangerous, and the migration of these to other bodily areas ${ }^{6}$.

\section{Influence of the gut microbiota on central nervous system}

In the recent literature, it has been demonstrated that the microbiota extends its influence not only at the level of the gastrointestinal tract but also at the level of the CNS with an important role in its functioning. The microbiota synthesizes neuroendocrine hormones capable of entering into communication both with the metasympathetic nervous system and the CNS. In fact, it has been found that bacteria not only have receptors for some neurotransmitters, but also are able themselves to produce substances such as noradrenaline, acetylcholine and serotonin. In this way, a bidirectional axis (gut-brain axis) is established between the intestine and the brain in which there is a synergistic communication between the "two brains" ( ref. $^{7}$ ). The bacteria, through the release of neurotransmitters, are therefore able to send signals at the CNS level and modulate the activity of the HPA axis thanks to the primary role of the vagus nerve. Therefore, the communication of the microbiota with the brain takes place through the vagus nerve, which allows the passage of information from the intestinal luminal environment to the CNS. Vagotomized mice lack these neurochemical and behavioral effects, identifying the vagus nerve as the main modulatory constitutive communication path between the microbiota and the brain ${ }^{8}$. The mechanisms that are established at the intestinal level to keep the gut-brain axis in equilibrium are the production and expression of neurotransmitters (serotonin, GABA) and neutrophic factors (BDNF); the protection of the intestinal barrier and the integrity of tight junctions; the production of bacterial metabolites; mucosal immune regulation and regulation of enteric sensory afferents ${ }^{7}$. An alteration at the bacterial level induces an imbalance in the stability of the brain-gut axis, which affects the nervous transmission of the whole organism by modeling the HPA axis. It is evident that the intestinal microbiota is necessary for the normal development of the HPA and that there is a phase, in early life, where colonization must occur to ensure normal development of the HPA axis ${ }^{9-11}$.

\section{The role of microbiota in a hypermeable intestine}

At intestinal level, the integrity of the intestinal epithelium that serves to maintain a condition of health is of primary importance. An alteration or a malfunction of the epithelium leads to intestinal hypertrophy, which is difficult to treat. The presence of eubiosis reduces the prob- ability of intestinal bacterial invasion in case of damage to the epithelial barrier through the release of inflammatory mediators $^{12}$ and immune mediators such as IL-17A, an interleukin involved in the differentiation of CD4 + cells $^{13}$. Bacterial alteration, with concomitant increase in LPS, negatively affect the function of the intestinal epithelial barrier $^{14}$.

\section{Intestinal dysbiosis in patients with fibromyalgia}

In the fibromyalgic patient, several studies have shown a condition of dysbiosis, that rather than being considered an aspect of FM can be considered a cause of the illness. In 2004, for the first time, researchers noticed that $100 \%$ (42/42) of the fibromyalgic patients included in their study, had small intestinal bacterial overgrowth (SIBO) noting that the degree of somatic pain in FMis significantly correlated with the hydrogen level of the breath test ${ }^{15}$. The increase in bacterial strains and their proliferation has been studied in other studies that have confirmed the relationship between alterations of the intestinal bacterial flora and the presence of the disease ${ }^{16,17}$. One might think, however, that dysbiosis is a consequence of FM and not one of the causes of the same, but, actually, it does not seem to be an effect. In fact, it has been noted that fibromyalgic patients with SIBO, after the eradication of small intestinal bacterial growth, have marked clinical improvements ${ }^{18}$. In addition to alteration of the intestinal microbiota, an altered intestinal permeability was also highlighted in patients with primary FM (ref. ${ }^{19}$ ). These patients had significantly higher plasma LPS levels than healthy individuals ${ }^{20}$. High LPS is associated with increase in $\operatorname{Ig} \mathrm{A}$ that is significantly related to disease severity and symptoms like muscle tension, fatigue, difficulties with concentration and memory. It shows that intestinal permeability causes a more pronounced immune response to LPS due to the different concentration of toxins in the bloodstream ${ }^{21,22}$. Bacterial species altered in the fibromyalgic patient have been identified. There is an abundance of species Faecalibacterium, Roseburia, Dorea, Coprococcus, Clostridium, Ruminococcus and Coprobacillus. The increase in these bacterial strains is also associated with an higher concentration of Bacteroides that has been identified as a specific marker for the diagnosis of myalgic encephalomyelitis/chronic fatigue syndrome (ME/CFS) without irritable bowel syndrome (IBS) (ref. ${ }^{23}$ ). In addition to the increase of some species there is a decrease in Firmicutes (such as Roseburia and Dialister), as has been noted in some studies ${ }^{24}$.

\section{Connection between dysbiosis and circulating hormones}

Several studies underline the relationship between particular types of bacteria in the intestine and the secretion of the hormones of the hypothalamus - hypophysis axis. Evaluating the composition of the microbiota and measuring the blood concentrations of the circulating hormones, it has been noted that blood levels of cortisol and serotonin are negatively correlated with the presence of Ruminococcus ${ }^{25}$. A negative correlation was also noted between the abundance of Coprococcus and the concen- 
tration of vitamin $\mathrm{D}$, whose presence in the circulation is also inversely proportional to the increase in LPS (ref. ${ }^{26}$ ). Thus, dysbiosis appears to affect the whole hormone system.

An altered intestinal flora decarboxes too much tryptophan in indole and skatole resulting in a decrease of tryptophan and therefore a minor its brain captation that reduces the synthesis of melatonin and serotonin. Finally, the correlation between dysbiosis and thyroid is suggested as a high concentration of LPS appears to be connected to decrease in thyroid hormones ${ }^{27}$ since the liposaccharide inhibits the enzyme that deals with the conversion from the inactive form of the hormone thyroxine (T4) to the active form called triiodothyronine (T3).

\section{Altered hormones and dysbiosis in fibromyalgic patients}

In the patient with fibromyalgia, altered hormonal secretion has been observed that could have a connection with the condition of dysbiosis. The alteration of the circadian cycle with the consequent sleep disorder, asthenia, muscle fatigue or weakness (the main symptoms of FM) represents conditions that are triggered after an altered hormone concentration. Several studies have noted that in the patient with FM there is a decrease in the concentration of cortisol, serotonin, vitamin D, thyroid hormones and melatonin and this could be a consequence of intestinal dysbiosis, as we will analyze below.

- Cortisol. Cortisol produced by the adrenal cortex, in addition to having numerous effects on glucose, lipid and protein metabolism plays a major role in resistance to stress and inflammation. The evidence of an inflammatory component in the patient with FM, debated for years, has been confirmed in several studies that have reported a high level of cytokines in the fibromyalgic patient showing inflammation in the central and peripheral nervous system ${ }^{28-30}$. In the patient with FM, the low level of cortisol $^{31,32}$ is therefore connected to a mild anti-inflammatory effect that causes a diffuse inflammatory condition. Based on what was discussed above, therefore, a lower level of cortisol in the circulation could be connected with a condition of dysbiosis in which there is an increase in the genus of Ruminococcus: both situations have been described in the patient with FM.

- Vitamin D. In the patient with FM, a lower concentration of vitamin D3 was noted ${ }^{33-35}$ and it appears to lead to an increase in chronic pain, one of the most serious symptoms of the disease. Vitamin D is able to reduce pain by releasing cytokines ${ }^{36}$ or reducing the sensitivity of nerve fibers in the muscles ${ }^{37,38}$. Vitamin $\mathrm{D}$ also appears to be involved in sleep modulation that is altered in the patient with FM (ref. ${ }^{39}$ ). Also in the case of vitamin D3, the connection between altered hormone secretion and dysbiosis in FM is therefore evident: the decrease in the concentration of vitamin D3 would be corrected with the increase in LPS, as highlighted above.

- Serotonin. In recent years there has been a marked interest on the possible involvement of serotonin in FM.
Serotonin (5-TH), synthesized from the amino acid tryptophan, plays a major role in sleep induction because administration of serotoninergic synthesis inhibitors causes persistent insomnia for days. Low levels of tryptophan and serotonin have been noted in patients with FM (ref. ${ }^{40,41}$ ) highlighting the correlation between low serotonin concentration and increased pain perception $^{41}$. The poor cerebral uptake of tryptophan and the consequent decrease in serotonin synthesis in FM could be due to excessive decarboxylation at the intestinal level, which involves an increase in indole and skatole and a decrease in the ratio trp/LNAAs (Trp: Tryptophane; LNAAs: LNAAs: Valine, Leucine, Isoleucine, Tyrosine, Phenylalanine) ( ref. $^{40,41}$ ).

- Thyroid hormones. An alteration of the concentration of thyroid hormones (T3, T4 and TSH) could be related to FM symptomatology. Dr. Lowe highlights the connection between thyroid disease and fibromyalgia. This is because thyroid dysfunction causes: fatigue (inadequate regulation of the thyroid hormone alters the amount of dopamine and its receptors) and excessive muscle tension because to completely relax the muscles, the filaments must lengthen and separate and this requires ATP which is reduced if there is a low level of thyroid hormones. Several studies have shown that in the fibromyalgic patient there is hypothyroidism of type II because there is a hormonal resistance ${ }^{42}$. Most cases of fibromyalgia fall into the latter category and therefore the connection between thyroid dysfunction and FM is evident, especially when there is an autoimmune problem: autoimmune thyroiditis is therefore present in a high percentage of FM patients and has been associated with the presence of typical symptoms of the disease $\mathrm{e}^{43}$. Although there is an evident connection between thyroid and FM the level of hormone can be normal or altered as II type iodases, responsible for the conversion of T4 in T3, appear to be down regulated in the patient with FM (ref. ${ }^{43}$ ). The altered FT3/FT4 ratio could, according to what has been discussed in the previous paragraphs, be connected with the disbiotic alteration in the fibromyalgic patient and specifically with the increase of LPS in the circulation.

- Melatonin. Melatonin, produced by the pineal gland, is a hormone involved in the regulation of the sleepwake cycle whose concentrations in the blood reaches a peak between 2 and 4 o'clock in the morning and then gradually decreases. The significance melatonin in FM is unclear. Some studies have noted a normal concentration $^{44}$, others a lower concentration ${ }^{45}$ and another has reported a greater level of hormone in circulation ${ }^{46}$. However, in the fibromyalgic patient it has been noted that melatonin is produced less at bedtime and this affects sleep and the circadian rhythm ${ }^{47}$. Administering melatonin in patients with FM, there was a beneficial effect on the general symptomato$\log y^{48}$ with less of pain also because disturbed nocturnal sleep in patients with FM causes pain, muscle stiffness and fatigue especially in the morning ${ }^{49}$. The decreased release of melatonin at bedtime may have 
as an etiology intestinal dysbiosis that - as already discussed in the previous paragraphs - causes less release of tryptophan and therefore decreased synthesis of the hormone.

\section{CONCLUSION}

This article, on the correlation between dysbiosis and altered hormone secretion in FM, draws attention to the role of the microbiota in the etiopathogenesis of FM from a new point of view. Regular physical activity, a healthy lifestyle, a diet rich in fiber with a moderate intake of fats and proteins and probiotics could rebalance the intestinal flora and reduce the symptoms of FM. Evaluating the altered bacterial flora in the patient with Fibromyalgia may be the key to reducing the severity of symptoms and improving the quality of life for patients with this debilitating disease. It is clear that further studies should be conducted to better assess the influence of the microbiota on hormonal alteration in the general FM population.

\section{Search strategy and selection criteria}

Our article focuses on the importance of microbiota as an etiological factor for extraintestinal diseases. The literature of the last 20 years was taken into consideration with particular reference to the years between 2011 and 2017. All the documents were searched through PubMed through keywords such as microbiota, cortisol, fibromyalgia, dysbiosis, vitamin D, thyroid hormones and serotonin.

Author contribution: All authors contributed equally to preparing the manuscript.

\section{REFERENCES}

1. Tomasello G, Mazzola M, Leone A, Sinagra E, Zummo G, Farina F, Damiani P, Cappello F, Gerges Geagea A, Jurjus A, Bou Assi T, Messina M, Carini F. Nutrition, oxidative stress and intestinal dys biosis: Influence of diet on gut microbiota in inflammatory bowel diseases. Biomed Pap Med Fac Univ Palacky Olomouc Czech Repub 2016;160(4):461-6.

2. Carini F, Sanfilippo A, Margiotta G, Mazzola M, Scardina GA, Messina M. Rappa F, Trovato E, Damiani P, Tomasello G. Inflammatory bowe disease and peripheral arthritis: mesalazina and probiotics. EMBJ 2016;11(15):112-7.

3. Mazzola M, Carini F, Leone A, Damiani P, Messina M, Jurjus A Tomasselo G. Ibd, malignancy and oral microbiota: analysis of the literature. Int J Clin Dent 2016;9(9),273-8.

4. Tomasello G, Mazzola M, Jurjus A, Cappello F, Carini F, Damiani $P$ Gerges Geagea A, Zeenny MN, Leone A. The fingerprint of the human gastrointestinal tract microbiota: a hypothesis of molecular mapping. J Biol Regul Homeost Agents 2017;31(1):245-9.

5. Mazzola M, Carini F, Leone A, Damiani P, Jurjus A, Geagea AG. Inflammatory bowel disease and colorectal cancer, nutraceutical aspects. EMBJ 2016;11,123-129.

6. Carini F, Tomasello G, Jurjus A, Geagea A, Al Kattar S, Damiani P, Sinagra E, Rappa F, David S, Cappello F, Mazzola M, Leone A. Colorectal cancer and inflammatory bowel diseases: effects of diet and antioxidants. J Biol Regul Homeost Agents 2017;31(3):791-5.

7. Carabotti M, Scirocco A, Maselli MA, Severi C. The gut-brain axis: in teractions between enteric microbiota, central and enteric nervous systems. Ann Gastroenterol 2015;28(2):203-9.

8. Bravo JA, Forsythe P, Chew MV, Escaravage E, Savignac HM, Dinan TG, Bienenstock J, Cryan JF. Ingestion of Lactobacillus strain regulates emotional behavior and central GABA receptor expression in a mouse via the vagus nerve. Proc Natl Acad Sci USA 2011;108(38):16050-5.

9. Sudo N, Chida Y, Aiba Y, Sonoda J, Oyama N, Yu XN, Kubo C, Koga Y. Postnatal microbial colonization programs the hypothalamicpituitary-adrenal system for stress response in mice. J Physiol 2004;558(Pt 1):263-75.

10. Cong X, Xu W, Romisher R, Poveda S, Forte S, Starkweather A, Henderson WA. Gut Microbiome and Infant Health: BrainGut-Microbiota Axis and Host Genetic Factors. Yale J Biol Med 2016;89(3):299-308.

11. Dinan TG, Cryan JF. Regulation of the stress response by the gut microbiota: implications for psychoneuroendocrinology. Psychoneuroendocrinology 2012;37(9):1369-78.

12. Scardina GA, Pisano T, Carini F, Valenza V, Messina P. Burning mouth syndrome: an evaluation of in vivo microcirculation. J Am Dent Assoc 2008;139(7):940-6.

13. Edelblum KL, Sharon G, Singh G, Odenwald MA, Sailer A, Cao $\mathrm{S}$. The Microbiome Activates CD4 T-cell-mediated Immunity to Compensate for Increased Intestinal Permeability. Cell Mol Gastroenterol Hepatol 2017;4(2):285-97.

14. Bein A, Zilbershtein A, Golosovsky M, Davidov D, Schwartz B. LPS Induces Hyper-Permeability of Intestinal Epithelial Cells. J Cell Physiol 2017;232(2):381-90.

15. Pimentel M, Wallace D, Hallegua D, Chow E, Kong Y, Park S, Lin HC. A link between irritable bowel syndrome and fibromyalgia may be related to findings on lactulose breath testing. Ann Rheum Dis 2004; 63(4):450-2.

16. Othman M, Agüero R, Lin HC. Alterations in intestinal microbial flora and human disease. Curr Opin Gastroenterol 2008;24(1):11-6.

17. Reichenberger ER, Alexander GM, Perreault MJ, Russell JA, Schwartzman RJ, Hershberg U, et al. Establishing a relationship between bacteria in the human gut and complex regional pain syndrome. Brain Behav Immun 2013;29:62-9.

18. Wallace DJ, Hallegua DS. Fibromyalgia: the gastrointestinal link. Curr Pain Headache Rep 2004;8(5):364-8.

19. Goebel A, Buhner S, Schedel R, Lochs H, Sprotte G. Altered intestinal permeability in patients with primary fibromyalgia and in patients with complex regional pain syndrome. Rheumatology (Oxford) 2008;47(8):1223-7.

20. Giloteaux L, Goodrich JK, Walters WA, Levine SM, Ley RE, Hanson MR. Reduced diversity and altered composition of the gut microbiome in individuals with myalgic encephalomyelitis/chronic fatigue syndrome. Microbiome 2016;4:30.

21. Maes M, Twisk FN, Kubera M, Ringel K, Leunis JC, Geffard M. Increased $\lg$ A responses to the LPS of commensal bacteria is associated with inflammation and activation of cell-mediated immunity in chronic fatigue syndrome. J Affect Disord 2012;136(3):909-17.

22. Maes M, Mihaylova I, Leunis JC. Increased serum IgA and IgM against LPS of enterobacteria in chronic fatigue syndrome (CFS): indication for the involvement of gram-negative enterobacteria in the etiology of CFS and for the presence of an increased gut-intestinal permeability. J Affect Disord 2007;99(1-3):237-40.

23. Nagy-Szakal D, Williams BL, Mishra N, Che X, Lee B, Bateman L, Klimas NG, Komaroff AL, Levine S, Montoya JG, Peterson DL, Ramanan D, Jain K, Eddy ML, Hornig M, Lipkin WI. Fecal metagenomic profiles in subgroups of patients with myalgic encephalomyelitis/chronic fatigue syndrome. Microbiome 2017;5(1):44.

24. Fremont M, Coomans D, Massart S, De Meirleir K. High-throughput 16S rRNA gene sequencing reveals alterations of intestinal microbiota in myalgic encephalomyelitis/chronic fatigue syndrome patients. Anaerobe 2013;22:50-6.

25. Mudd AT, Berding K, Wang M, Donovan SM, Dilger RN. Serum cortiso mediates the relationship between fecal Ruminococcus and brain $\mathrm{N}$-acetylaspartate in the young pig. Gut Microbes 2017;8(6):589-600.

26. Luthold RV, Fernandes GR, Franco-de-Moraes AC, Folchetti LG, Ferreira SR. Gut microbiota interactions with the immunomodulatory role of vitamin D in normal individuals. Metabolism 2017;69:7686. 
27. Boelen A, Kwakkel J, Platvoet-ter Schiphorst M, Mentrup B, Baur A, Koehrle J, Wiersinga WM. Interleukin-18, a proinflammatory cytokine, contributes to the pathogenesis of non-thyroidal illness mainly via the central part of the hypothalamus-pituitary-thyroid axis. Eur J Endocrinol 2004;151(4):497-502.

28. Bote ME, García JJ, Hinchado MD, Ortega E. Inflammatory/ stress feedback dysregulation in women with fibromyalgia. Neuroimmunomodulation 2012;19(6):343-51.

29. Cordero MD, Díaz-Parrado E, Carrión AM, Alfonsi S, Sánchez-Alcazar $J A$, Bullón $P$, Battino $M$, de Miguel M. Is inflammation a mitochondrial dysfunction-dependent event in fibromyalgia? Antioxid Redox Signal 2013;18(7):800-7.

30. Bäckryd E, Tanum L, Lind AL, Larsson A, Gordh T. Evidence of both systemic inflammation and neuroinflammation in fibromyalgia patients, as assessed by a multiplex protein panel applied to the cerebrospinal fluid and to plasma. J Pain Res 2017;10:515-25. doi: 10.2147/JPR.S128508

31. Riva R, Mork PJ, Westgaard RH, Rø M, Lundberg U. Fibromyalgia syndrome is associated with hypocortisolism. Int J Behav Med 2010;17(3):223-33.

32. Semiz EA, Hizmetli S, Semiz M, Karadağ A, Adalı M, Tuncay MS, Alim B, Hayta E, Uslu AU. Serum cortisol and dehydroepiandrosteronesulfate levels after balneotherapy and physical therapy in patients with fibromyalgia. Saudi Med J 2016;37(5):544-50.

33. Matthana MH. The relation between vitamin D deficiency and fibromyalgia syndrome in women. Saudi Med J 2011;32(9):925-9.

34. Abokrysha NT. Vitamin D deficiency in women with fibromyalgia in Saudi Arabia. Pain Med 2012;13(3):452-8.

35. Makrani AH, Afshari M, Ghajar M, Forooghi Z, Moosazadeh M. Vitamin D and fibromyalgia: a meta-analysis. Korean J Pain. 2017;30(4):250-7.

36. Azizieh F, Alyahya KO, Raghupathy R. Association between levels of vitamin $\mathrm{D}$ and inflammatory markers in healthy women. J Inflamm Res 2016;9:51-7.

37. Tague SE, Clarke GL, Winter MK, McCarson KE, Wright DE, Smith PG. Vitamin D deficiency promotes skeletal muscle hypersensitivity and sensory hyperinnervation. J Neurosci 2011;31(39):13728-38.

38. Tague SE, Smith PG. Vitamin D deficiency leads to sensory and sympathetic denervation of the rat synovium. Neuroscience 2014; 279:77-93.

39. Piovezan RD, Hirotsu C, Feres MC, Cintra FD, Andersen ML, Tufik S, Poyares D. Obstructive sleep apnea and objective short sleep duration are independently associated with the risk of serum vitamin $D$ deficiency. PLoS One 2017;12(7):e0180901.

40. Yunus M B, Dailey J W, Aldag J C. Plasma tryptophan and other amino acids in primary fibromyalgia: a controlled study. J Rheumato 1992;19(1):90-4.

41. Hrycaj P, Stratz T, Muller W. Platelet $3 \mathrm{H}$-imipramine uptake receptor density and serum serotonin levels in patients with fibromyalgia/ fibrositis syndrome. J Rheumatol 1992;19(1):104-9.

42. Garrison RL, Breeding PC. A metabolic basis for fibromyalgia and its related disorders: the possible role of resistance to thyroid hormone Med Hypotheses 2003;61(2):182-9.

43. Bazzichi L, Rossi A, Giuliano T, De Feo F, Giacomelli C, Consensi A Ciapparelli A, Consoli G, Dell'Osso L, Bombardieri S. Association between thyroid autoimmunity and fibromyalgic disease severity. Clin Rheumatol 2007;26(12):2115-20.

44. Press J, Phillip M, Neumann L, Barak R, Segev Y, Abu-Shakra M, Buskila D. Normal melatonin levels in patients with fibromyalgia syndrome. J Rheumatol 1998;25(3):551-5.

45. Wikner J, Hirsch U, Wetterberg L, Rodjmark S. Fibromyalgia: a syndrome associated with decreased nocturnal melatonin secretion. Clin Endocrinol 1998;49,179-83.

46. Korszun A, Sackett-Lundeen L, Papadopoulos E. Melatonin levels in women with fibromyalgia and chronic fatigue syndrome. $J$ Rheumatol 1999;26,2675-80.

47. Mahdi AA, Fatima G, Das SK, Verma NS. Abnormality of Circadian Rhythm of Serum Melatonin and Other Biochemical Parameters in Fibromyalgia Syndrome. Indian J Biochem Biophys 2011;48(2):82-7.

48. Citera G, Arias MA, Maldonado-Cocco JA, Lázaro MA, Rosemffet MG, Brusco LI, Scheines EJ, Cardinalli DP. The effect of melatonin in patients with fibromyalgia: a pilot study. Clin Rheumatol 2000;19(1):913.

49. Hussain SA, Al-Khalifa II, Jasim NA, Gorial FI. Adjuvant use of melatonin for treatment of fibromyalgia. J Pineal Res 2011;50(3):267-71. 